\title{
MIMO INTERFERENCE AND PATH LOSS REDUCTION USING ADAPTIVE ANTENNA SYSTEM ALONG WITH OPTIMIZATION OF FREQUENCY FOR ZERO PATH LOSS
}

\author{
Aritra De $^{1^{*}}$ and Tirthankar Datta ${ }^{2}$ \\ ${ }^{1}$ Techno International Batanagar \\ ${ }^{2}$ Netaji Subhash Engineering College \\ ${ }^{1}$ Dearitra2001@yahoo.co.in, ${ }^{2}$ ask.tdatta@gmail.com
}

\begin{abstract}
Wireless communication technology is used by almost everyone now a day, so a large number of users can be handled by the wireless communication system where Multiple Input \& Multiple Output (MIMO)technology is used. The main problems of MIMO are interference due to a large number of users and path loss due to traverse of information or signal through the medium. The solutions of abovementioned problems are discussed here. The path loss is calculated at the receiver by standard model and the inverse of those attenuations are calculated and path losses are eliminated at the receiver by Adaptive Antenna Technology (AAT). The attenuation is also reduced by optimize slope method (OSM) where attenuation is eliminated by maintaining a frequency to distance ratio by varying the frequency. $B y$ using standard CDMA technology, the problem of interference is also addressed.
\end{abstract}

Keywords- AAT, CDMA, MIMO, OSM, SISO

\section{INTRODUCTION}

Wireless communication performance can be improved by adopting Multiple Input Multiple Output (MIMO) technology which is also known as spatial diversity [1]. MIMO can handle a large number of users which is in demand of modern wireless communication technology [1]. The major disadvantages of MIMO are interference due to a large number of users and path loss of the medium in which the signals are transmitted. The interference problem can be addressed by adopting Code Division Multiple Access Technology (CDMA) [2]. CDMA uses different codes for different users. However, the problem of path loss, also known as fading is very difficult to remove [3]. The fading is two types large scale fading (when there is a direct path between transmitter and receiver is known as Line of Sight or LOS) and small-scale fading (when there is no direct path between transmitter and receiver is known as Non-Line of Sight or NLOS). If the fading is removed then signal quality at the receiver is improved. The interference or fading problem removal is technology is explained in this research work.

In 1996 Foschini pointed out [4] that significant system capacity will be enhanced with the use of multiple antennas at the transmitter and at the receiver. Large diversity gains are desirable to reduce the effect of fading. Thus, multipath propagation should be exploited whenever it exists [5]. Adding multipath components does not automatically lead to better performance [6-8]. For practical receiver designs multipath always lead to serious multiple access and self-interference, reducing the effective system capacity [9].

Received: November 26, 2018

Reviewed: January 28, 2019

Accepted: February 4, 2019

* Corresponding Author 
A general MIMO model which are used by 8 users is considered [10-12]. The information of all the users at the transmitter (Tx) are cascaded and transmitted through the medium and at receiver $(\mathrm{Rx})$ there eight number of users are considered. A predefined attenuation model is considered in this research work. The path loss in the medium is modelled by curve fitting method where a set of data is taken in a frequency range of 800$2000 \mathrm{MHz}$ and base station antenna heights $50 \mathrm{~m}$ over a distance of $5 \mathrm{~km} \mathrm{[13].}$

The paper is arranged as follows: The system model of the transmitter (Tx) and receiver (Rx)is considered in section II. analysis of performance is described in Section III results and discussions are shown in Section IV, the conclusion is described in Section V.

\section{SYSTEM MODEL}

The transmitter (Tx) is depicted in Figure 1, where 8 users are cascaded and transmitted through the medium. The path loss which is known as attenuation or fading is denoted by alpha $(\alpha)$ is introduced in the medium. A different code is used by the individual user which is known as CDMA. Delay spread multipath environments and electromagnetic interference are a very common problem in mobile communication. In a multiuser environment, wireless transmission performance is fundamentally limited by interference due to the presence of signals from other users as well as from multipath.

One of the solutions is the CDMA technology. Scientists have tried to develop a wireless system which can work satisfactorily, even under strong interference condition under CDMA technique. The high-power jamming problem beyond Direct Sequence Spread Spectrum [DSSS] jamming margin is solved by utilizing frequency diversion technique of the carrier frequency. So, the Spread Spectrum technology along with CDMA (code division multiple access) provides a solution for multiple access with its reduced interference level. But the Co-channel interference is suppressed in the CDMA system satisfactory up to the jamming margin level above which it is prone to interference. This may be the situation when the cars stop at the crossing of roads and the mobile units at the cars face infinite dwell time. Additionally, the limitations of data rate arise from the different speed of the user. The higher data rate is well supported for the user under low mobility condition like walking etc. But while moving in a high-speed vehicle, the multipath fading is accentuated, again at high data rate multipath distortion is more disturbing at a high data rate which in turn degrades the quality of service.

The model which is considered in this research work direct path between the transmitter (Tx) and receiver $(\mathrm{Rx})$ is considered as direct path carry more power and less attenuation due to less reflection and refraction.

The receiver is described in Figure 2 where a replica of the transmitted signal is received by eight receivers and multiplied by a weight which is the inverse matrix of attenuation matrix was calculated by the existing standard model. This method is named as Adaptive Antenna Technology (AAT).
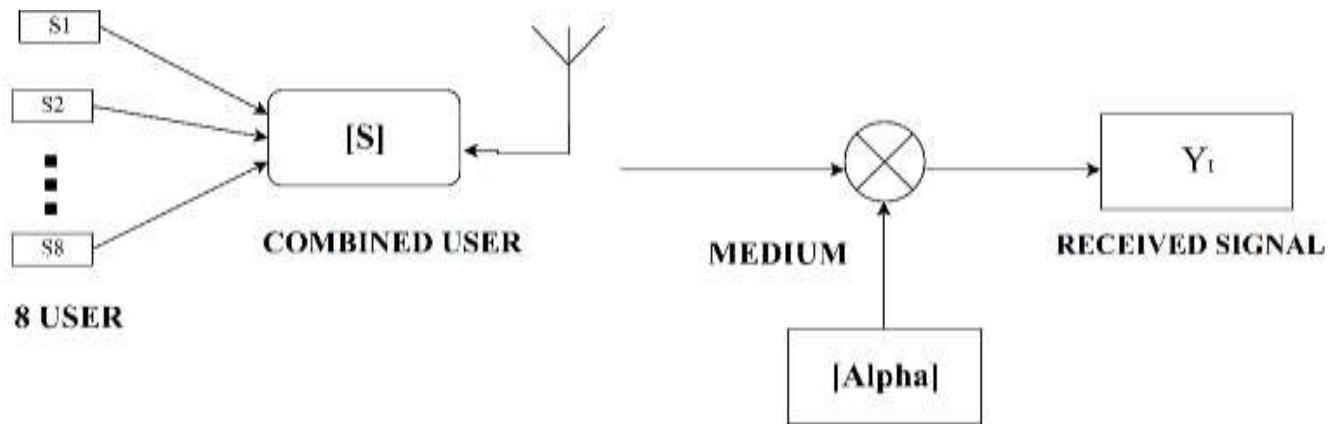

Fig. 1 Transmitter Model of The System 


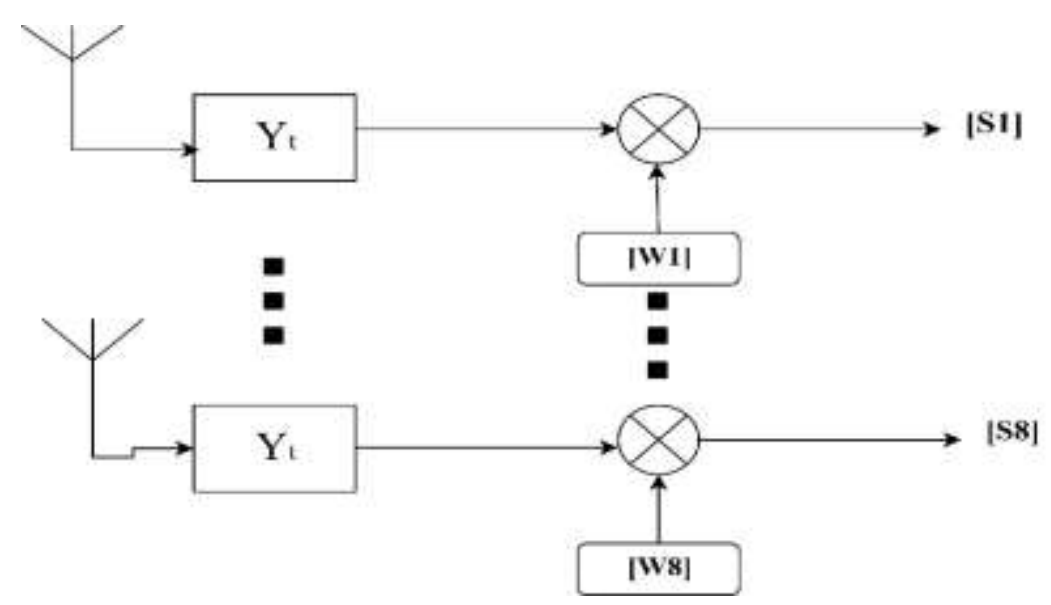

Fig. 2 Receiver(Rx) Model of The System

The path loss also can be reduced by another technology where the negative slope of frequency to distance is maintained a constant ratio which is named Optimization Slope Method (OSM).

\section{ANALYSIS OF PERFORMANCE}

Adaptive Antenna Technology (AAT) system plays an important role in the mitigation of multipath effects. The capacity improvement of the wireless network has drawn considerable attention to multiple-input-multiple-output (MIMO) system. Multiple antenna arrays at both the transmitter and receiver side of a communication link significantly improve the capacity over the single antenna system. Smart antenna is used widely to mitigate CCI.

An important research area for the antenna system is the processing block which consists of weight. In this work, the weights are calculated and multiplying with the incoming signal to get only the desired signal.

In this work, the weights are calculated and multiplying with the incoming signal to get only the desired signal. Here 8 mobile units and 8 antennas are considered at the receiver (Rx) station. In order to substantiate our present model, the cell size of the $1 \mathrm{~km}$ radius and the transmitting power of 1 watt are considered.

For unity gain the free space loss $(\alpha)$ expressed in $\mathrm{dB}$ as

$$
\alpha=(32.4+20 * \log (\mathrm{d})+20 * \log (\mathrm{f}))
$$

where $\mathrm{d}$ is the distance in kilometre and $\mathrm{f}$ is the carrier frequency in $\mathrm{MHz}$ Here at the 900 $\mathrm{MHz}$ frequency band is considered, however, it can be calculated for $2.4 \mathrm{GHz}$ also. However, for different areas, this equation will deviate from the present form.

The signal matrix is considered as below

$\mathrm{S}=\left[S_{1} S_{2} S_{3} S_{4} S_{5} S_{6} S_{7} S_{8}\right]$

$$
=\left[\begin{array}{ll}
29.830 .0 & 31.2 \\
28 & 30.729 .42930 .6
\end{array}\right]
$$

The corresponding distance matrix is $\mathrm{d}$ is given by

$\mathrm{d}=$ The corresponding attenuation matrix is calculated from equation 1 .

So, the transmitted signal is given by as shown in figure 1 is

$$
\left[Y_{t}\right]=[S]_{1 \times 8} *[\alpha]_{8 \times 8}
$$

The weight $\mathrm{W}$ is calculated by the inverse of matrix $\alpha$ $[W]_{8 \times 8}=\operatorname{inverse}(\alpha)$

$$
=\left[W_{i}\right]
$$


Where $\mathrm{i}$ is varied from 1 to 8 and each $W_{i}$ is an $8 \times 1$ matrix So, at the receiver signals are received by

$$
S_{i}=[S]_{1 \times 8} *[\alpha]_{8 \times 8} *\left[W_{i}\right]_{8 \times 1}
$$

Now to calculate the slope for zero attenuation derivative of equation (1) with respect to $\mathrm{d}$ is given by

$$
\frac{d \alpha}{d d}=\frac{20}{\ln (10)}\left[\frac{1}{d}+\frac{1}{f} * \frac{d f}{d d}\right]
$$

Now if zero is equated with equation (6), then $\frac{d f}{d d}$ is given by

$$
\frac{d f}{d d}=-\left(\frac{f}{d}\right)
$$

Hence from the equation (7) it is clear that for zero attenuation the slope value must be maintained.

\section{RESULTS AND DISCUSSIONS}

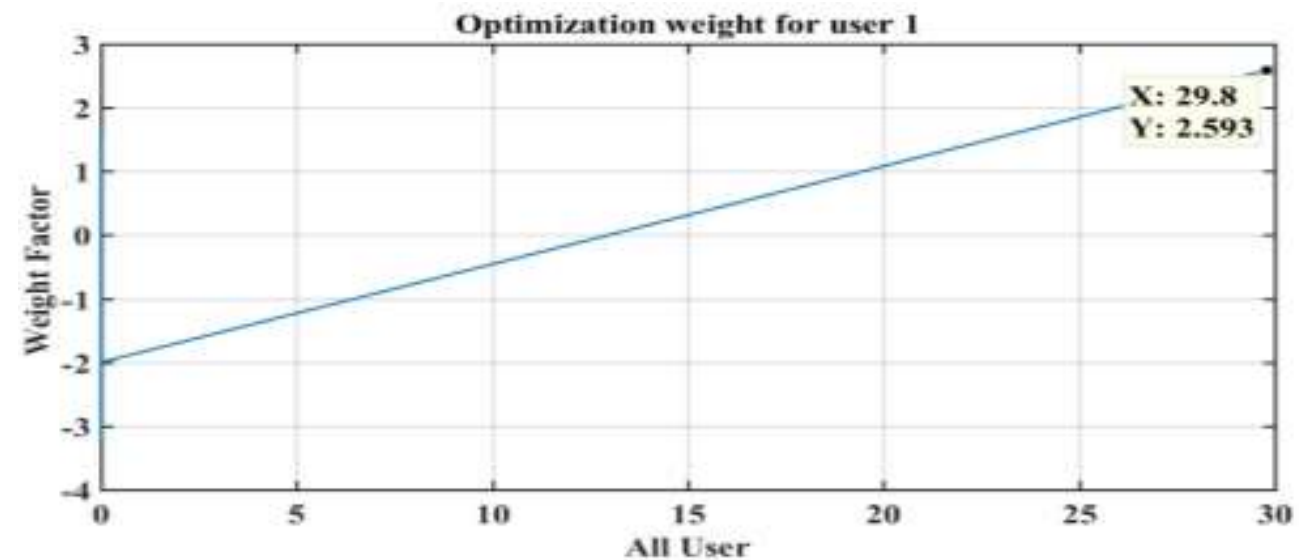

Fig. 3 Optimization Weight for User 1

The optimized weight value for user 1 is given by:

$W_{1}=[2.5929 ;-1.9960 ;-0.5079 ;-0.1150 ; 1.5548 ; 1.2178 ; 0.4298 ;-3.2061]$. It's the inverse matrix of the attenuation thus cancel the attenuation matrix (which can be predicted from standard model value) of the medium and the optimum weight across the user 1 is 2.593 then there is no attenuation and exact information of the user can be detected across the receiver.

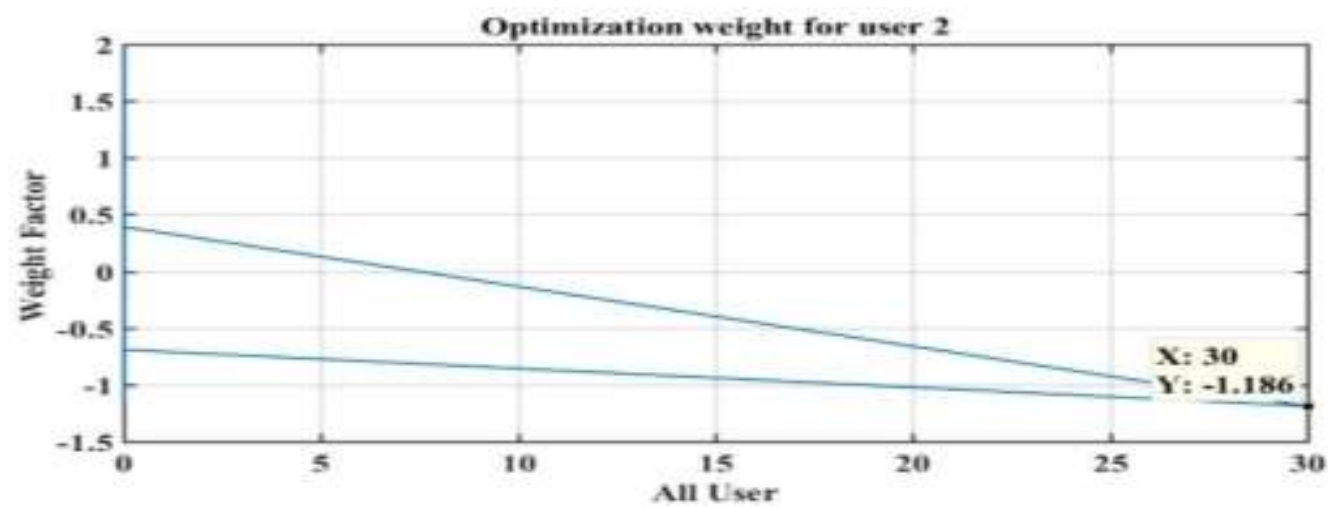

Fig. 4 Optimization Weight for User 2 
The optimized weight value for user 2 is given by:

$W_{2}=[-0.6892 ;-1.1864 ; 0.3935 ; 0.6711 ;-1.1515 ;-0.4686 ; 0.4988 \quad 1.9951$ It's the inverse matrix of the attenuation thus cancel the attenuation matrix (which can be predicted from standard model value) of the medium and the optimum weight across the user 2 is -1.186 then there is no attenuation and exact information of the user can be detected across the receiver.

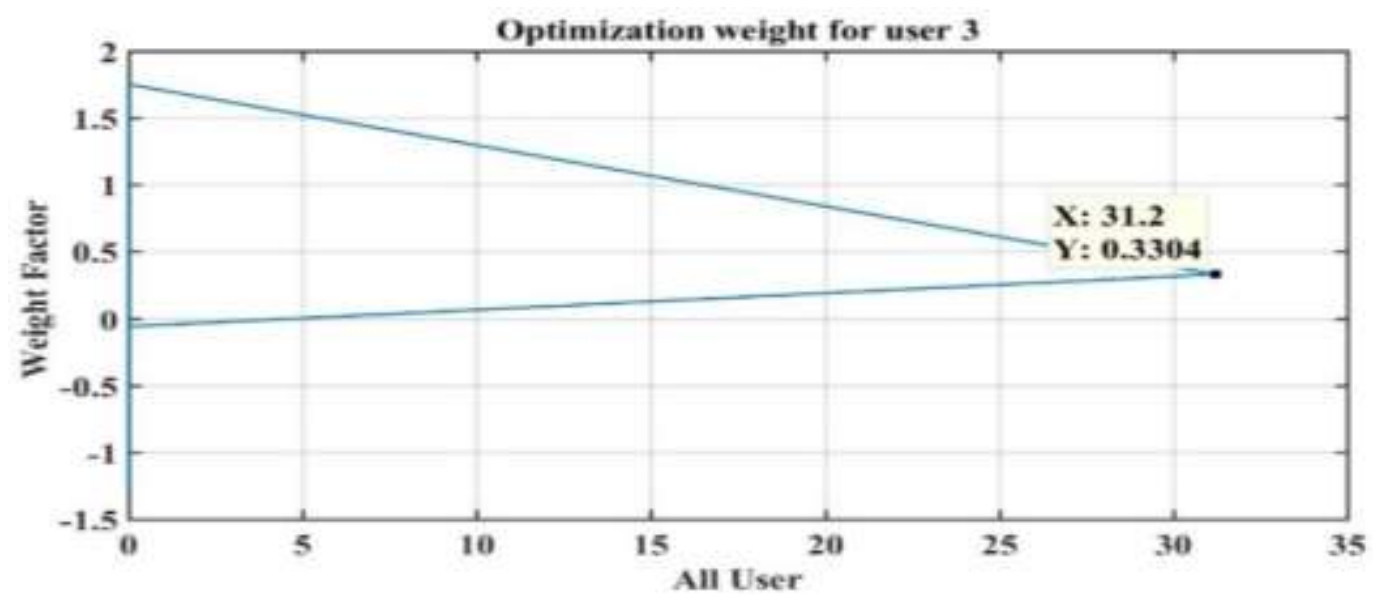

Fig. 5 Optimization Weight for User 3

The optimized weight value for user 3 is given by:

$W_{3}=[-1.1521 ; 1.7516 ; 0.3304 ;-0.0592 ;-1.2698 ;-0.4183 ;-0.32131 .1577]$. It's the inverse matrix of the attenuation thus cancel the attenuation matrix (which can be predicted from standard model value) of the medium and the optimum weight across the user 3 is 0.3304 then there is no attenuation and exact information of the user can be detected across the receiver.

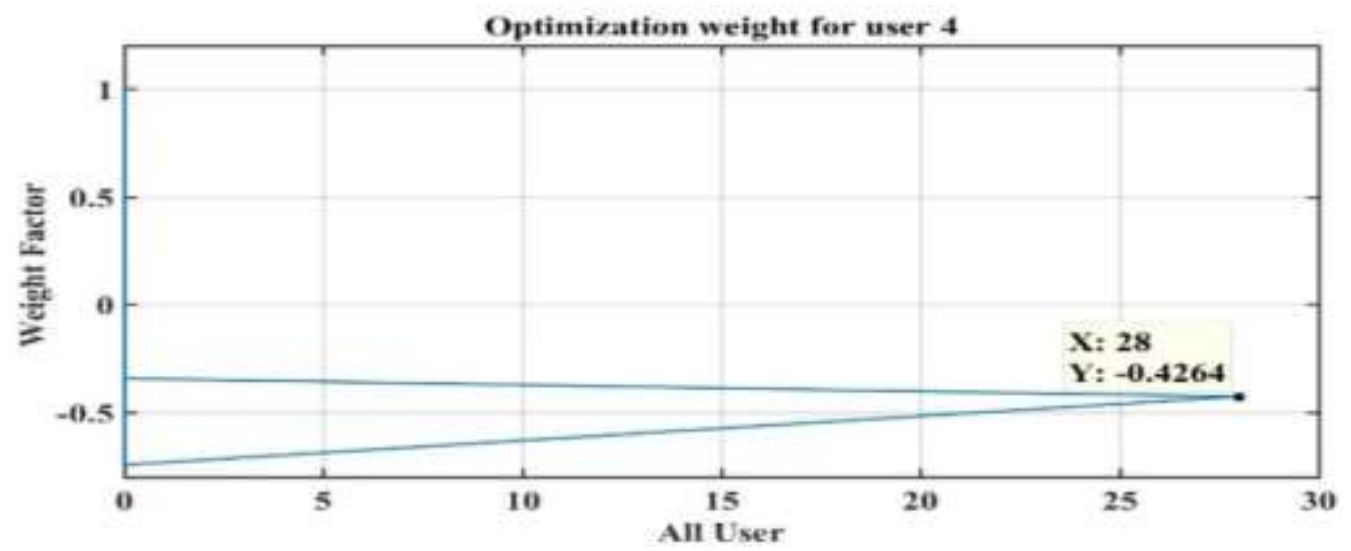

Fig. 6 Optimization Weight for User 4

The optimized weight value for user 4 is given by:

$W_{4}=[1.0298 ; 0.6927 ;-0.7433 ;-0.4264 \quad-0.3422 \quad-0.4006 \quad-0.4942 \quad 0.6519]$. It's the inverse matrix of the attenuation thus cancel the attenuation matrix (which can be predicted from standard model value) of the medium and the optimum weight across the user 4 is 0.4264 then there is no attenuation and exact information of the user can be detected across the receiver. 


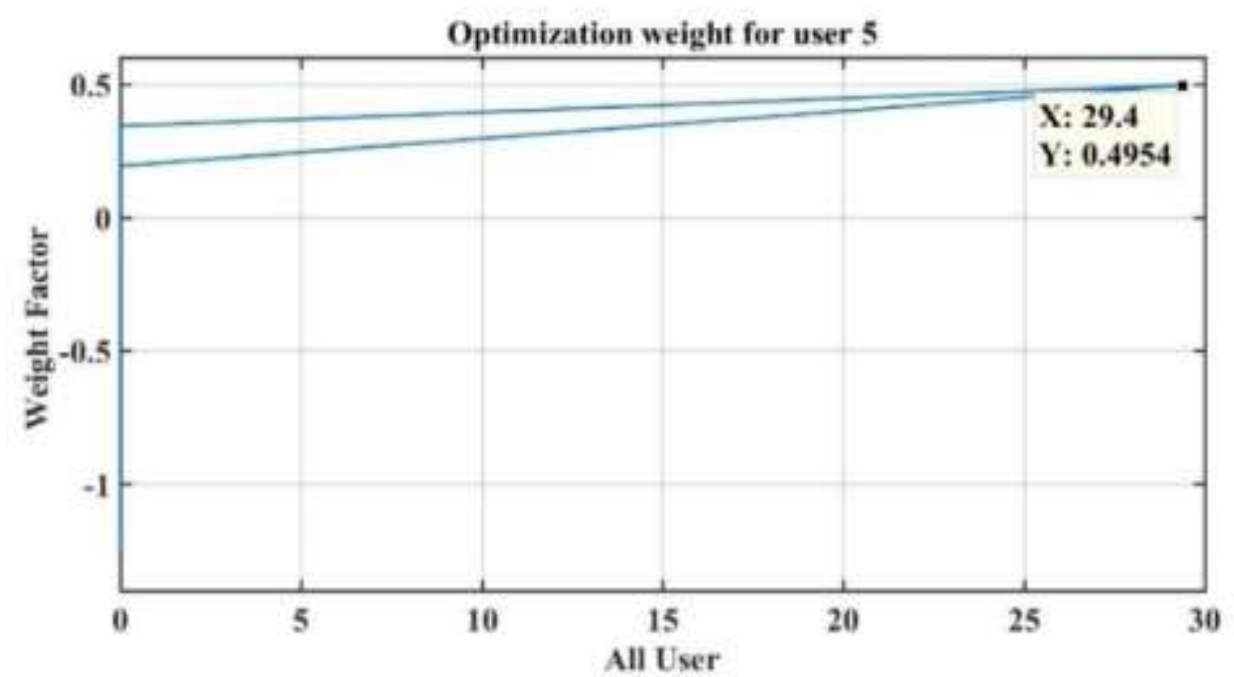

Fig. 7 Optimization Weight for User 5

The optimized weight value for user 5 is given by:

$W_{5}=[-1.2599 ; 0.3586 ;-0.0050 ; 0.1923 ; 0.4954 ; 0.3420 ; 0.1751 ;-0.2737]$. It's the inverse matrix of the attenuation thus cancel the attenuation matrix (which can be predicted from standard model value) of the medium and the optimum weight across the user 5 is 0.4954 then there is no attenuation and exact information of the user can be detected across the receiver.

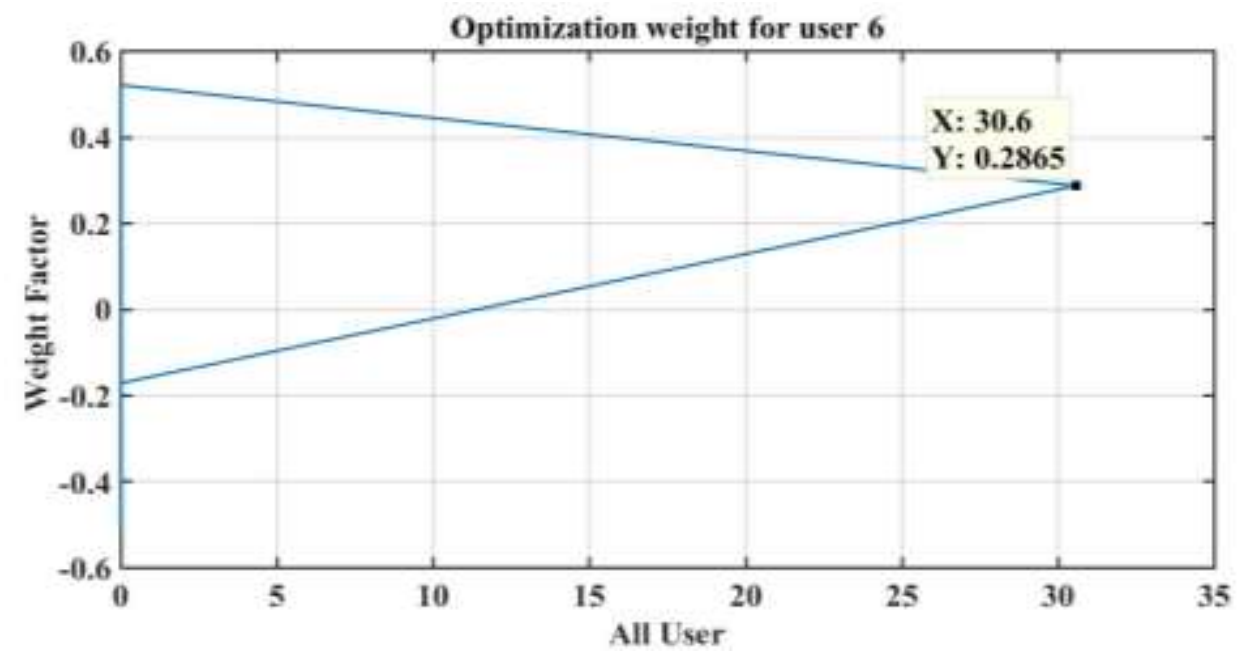

Fig. 8 Optimization Weight for User 6

The optimized weight value for user 6 is given by:

$W_{6}=[0.1181 ;-0.4887 ; 0.1374 ; 0.1195 ; 0.5203 ; 0.2865 ;-0.1719 ;-0.5139$ It's the inverse matrix of the attenuation thus cancel the attenuation matrix (which can be predicted from standard model value) of the medium and the optimum weight across the user 6 is 0.2865 then there is no attenuation and exact information of the user can be detected across the receiver. 


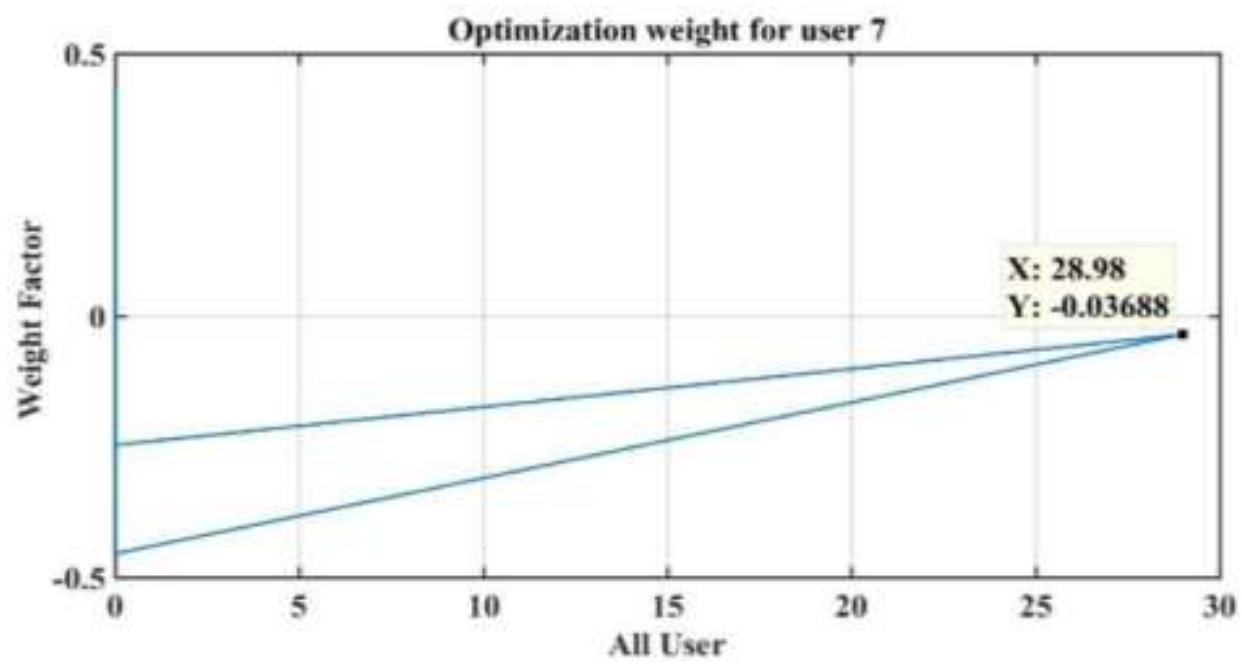

Fig. 9 Optimization Weight for User 7

The optimized weight value for user 7 is given by:

$W_{7}=[-0.2549 ; 0.4329 ; 0.2768 ;-0.0014 ; 0.2929 ;-0.4550 ;-0.0369-0.2477$ It's the inverse matrix of the attenuation thus cancel the attenuation matrix (which can be predicted from standard model value) of the medium and the optimum weight across the user 7 is -0.03688 then there is no attenuation and exact information of the user can be detected across the receiver.

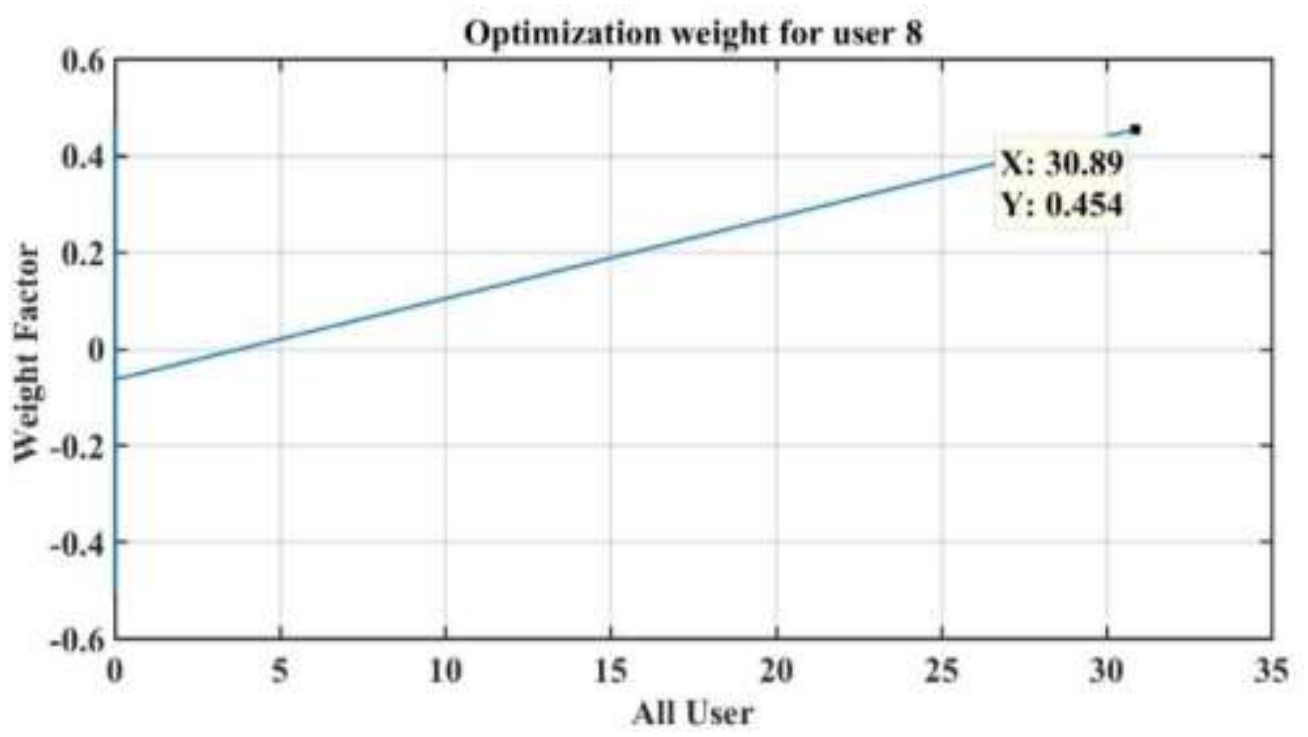

Fig. 10 Optimization Weight for User 8

The optimized weight value for user 8 is given by:

$W_{8}=[-0.5093 ; 0.4707 ; 0.2005 ;-0.3614 ;-0.1090 ;-0.0664 ;-0.06470 .4540]$. It's the inverse matrix of the attenuation thus cancel the attenuation matrix (which can be predicted from standard model value) of the medium and the optimum weight across the user 8 is 0.454 then there is no attenuation and exact information of the user can be detected across the receiver. 


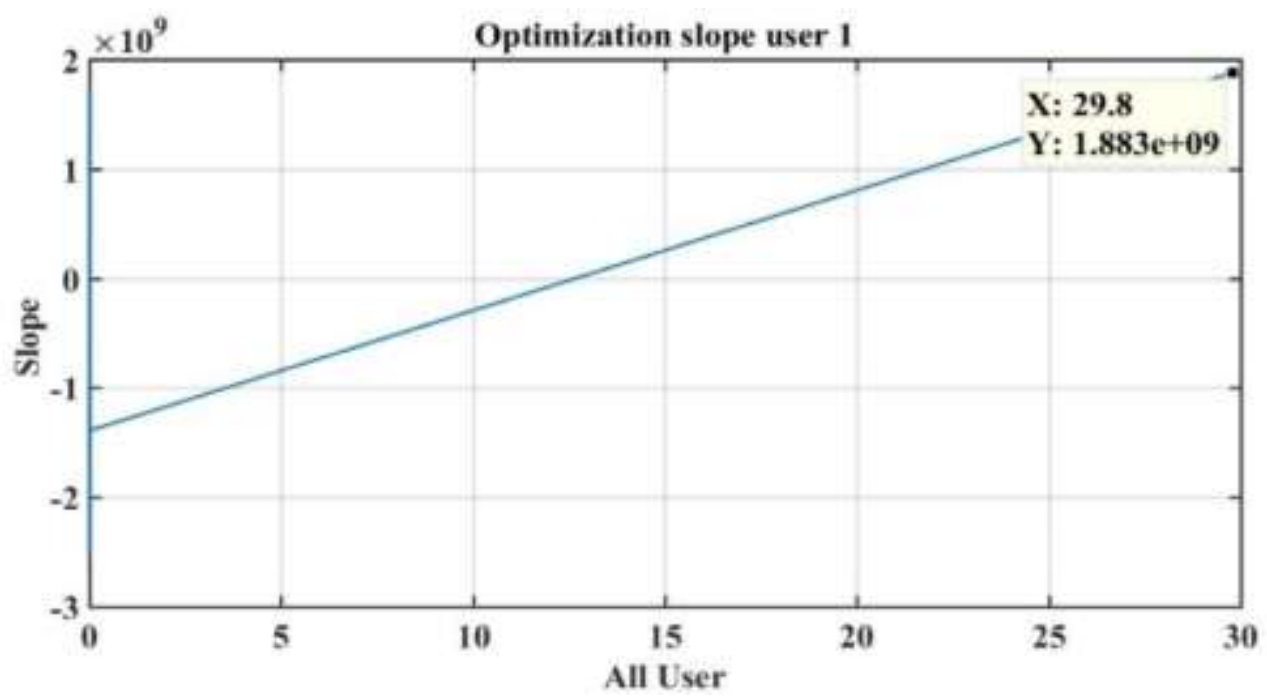

Fig. 11 Optimization Slope for User 1

The optimization slope for user 1 is given by:

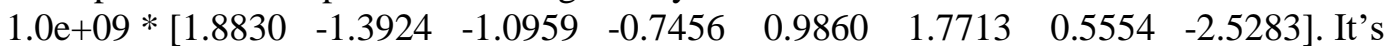
the value of slope(frequency/distance) which is maintained to cancel attenuation due to distance and the optimum value across user 1 is $1.0 \mathrm{e}+09 * 1.883$ then there is no attenuation and exact information of the user can be detected across the receiver.

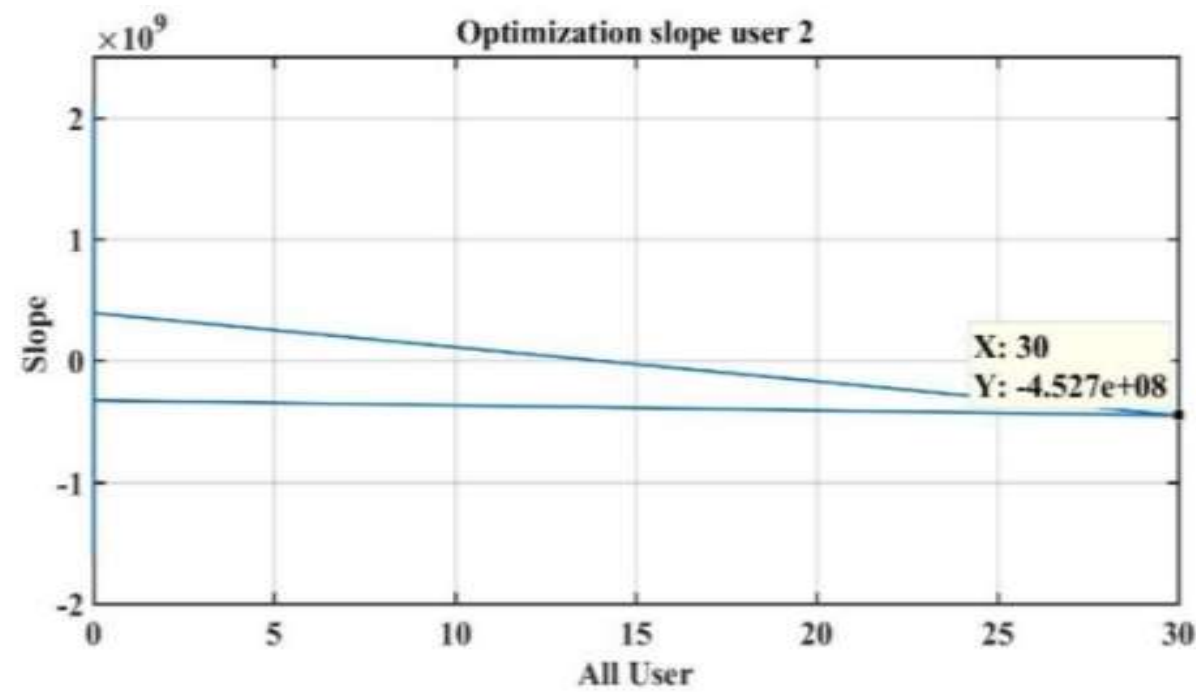

Fig. 12 Optimization Slope for User 2

The optimization slope for user 2 is given by:

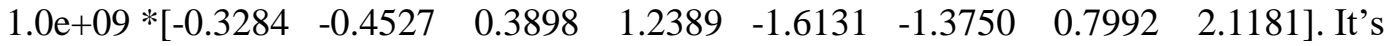
the value of slope(frequency/distance) which is maintained to cancel attenuation due to distance and the optimum value across user 2 is $1.0 \mathrm{e}+09 *(-4.527)$ then there is no attenuation and exact information of the user can be detected across the receiver. 


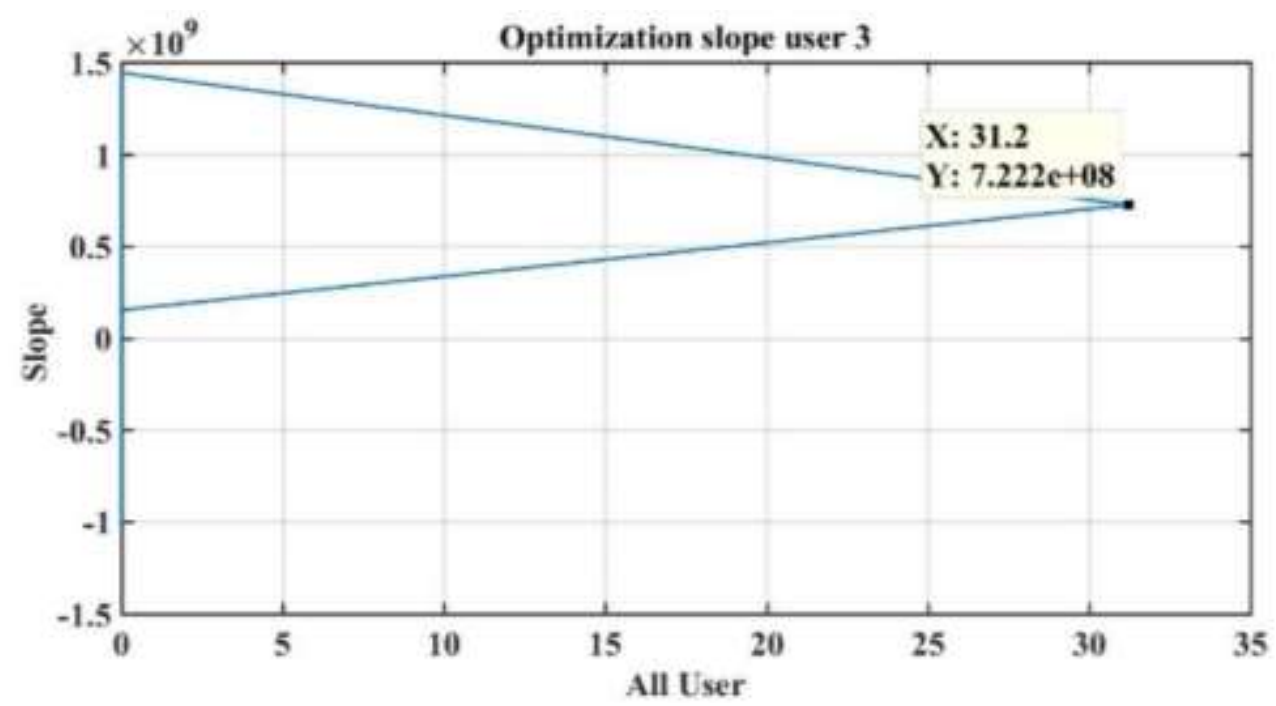

Fig. 13 Optimization Slope for User 3

The optimization slope for user 3 is given by:

$1.0 \mathrm{e}+09 *\left[\begin{array}{llllllll}-0.7307 & 1.4412 & 0.7222 & 0.1515 & -1.0184 & -0.4470 & -0.4221 & 0.7544\end{array}\right]$. It's the value of slope(frequency/distance) which is maintained to cancel attenuation due to distance and the optimum value across user 3 is $1.0 \mathrm{e}+09 *(7.222)$ then there is no attenuation and exact information of the user can be detected across the receiver.

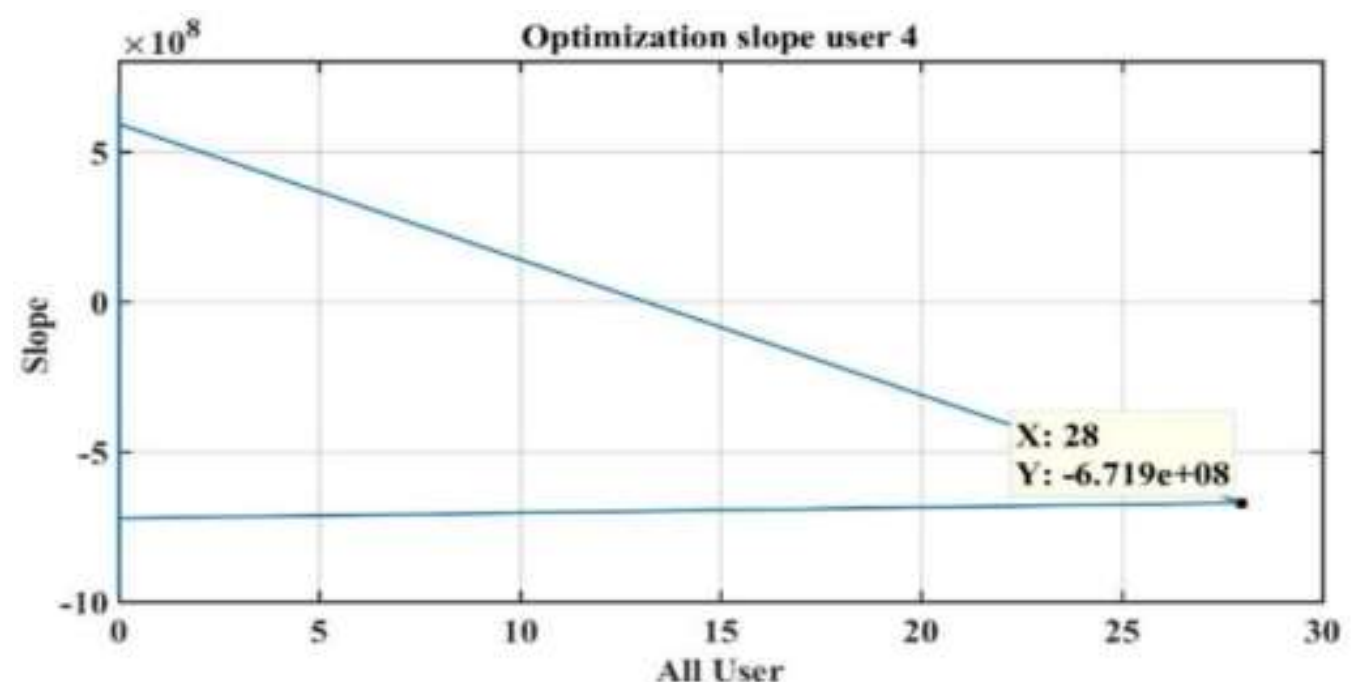

Fig. 14 Optimization Slope for User 4

The optimization slope for user 4 is given by:

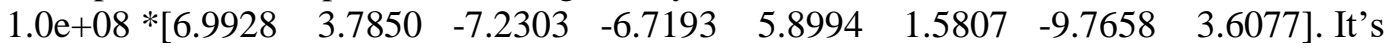
the value of slope(frequency/distance) which is maintained to cancel attenuation due to distance and the optimum value across user 4 is $1.0 \mathrm{e}+09 *(--6.719)$ then there is no attenuation and exact information of the user can be detected across the receiver. 


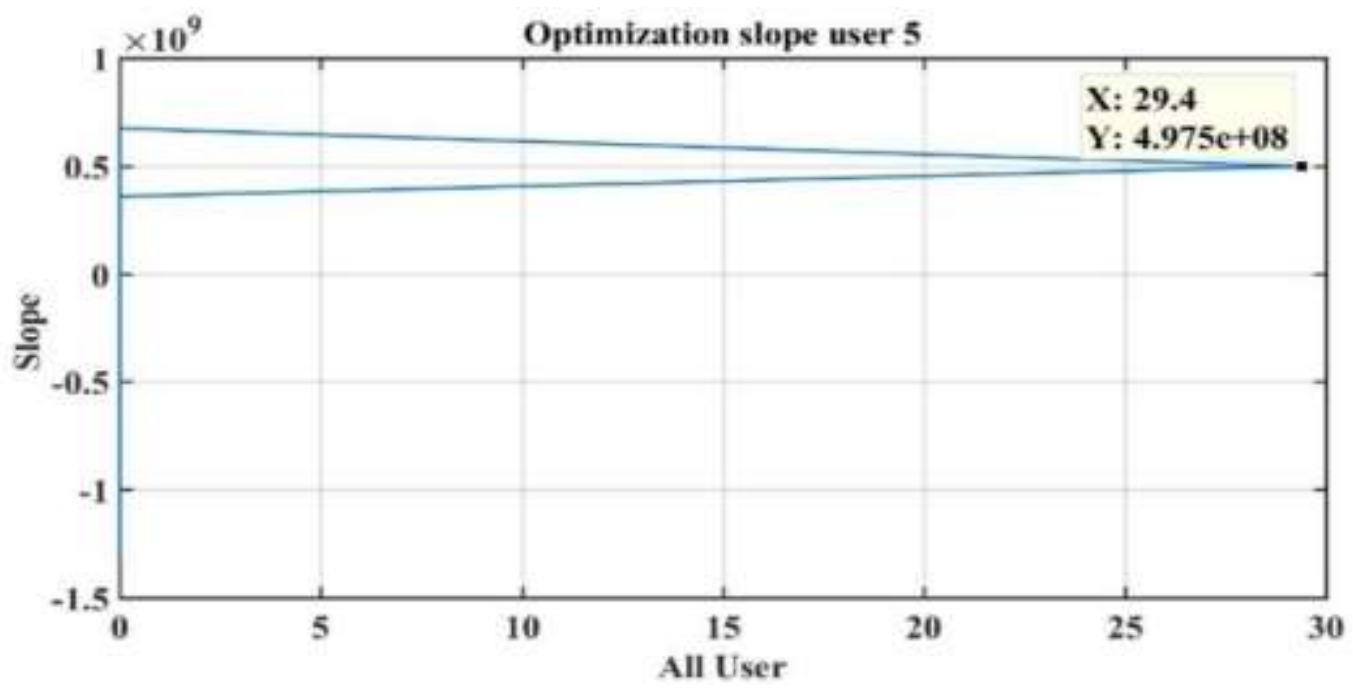

Fig. 15 Optimization Slope for User 5

The optimization slope for user 5 is given by: $1.0 \mathrm{e}+09 *\left[\begin{array}{llllllll}-1.3301 & 0.4060 & -0.7427 & 0.3583 & 0.4975 & 0.6740 & 0.6222 & -0.1546] . ~ I t ' s\end{array}\right.$ the value of slope(frequency/distance) which is maintained to cancel attenuation due to distance and the optimum value across user 5 is $1.0 \mathrm{e}+09 * 4.975$ then there is no attenuation and exact information of the user can be detected across the receiver.

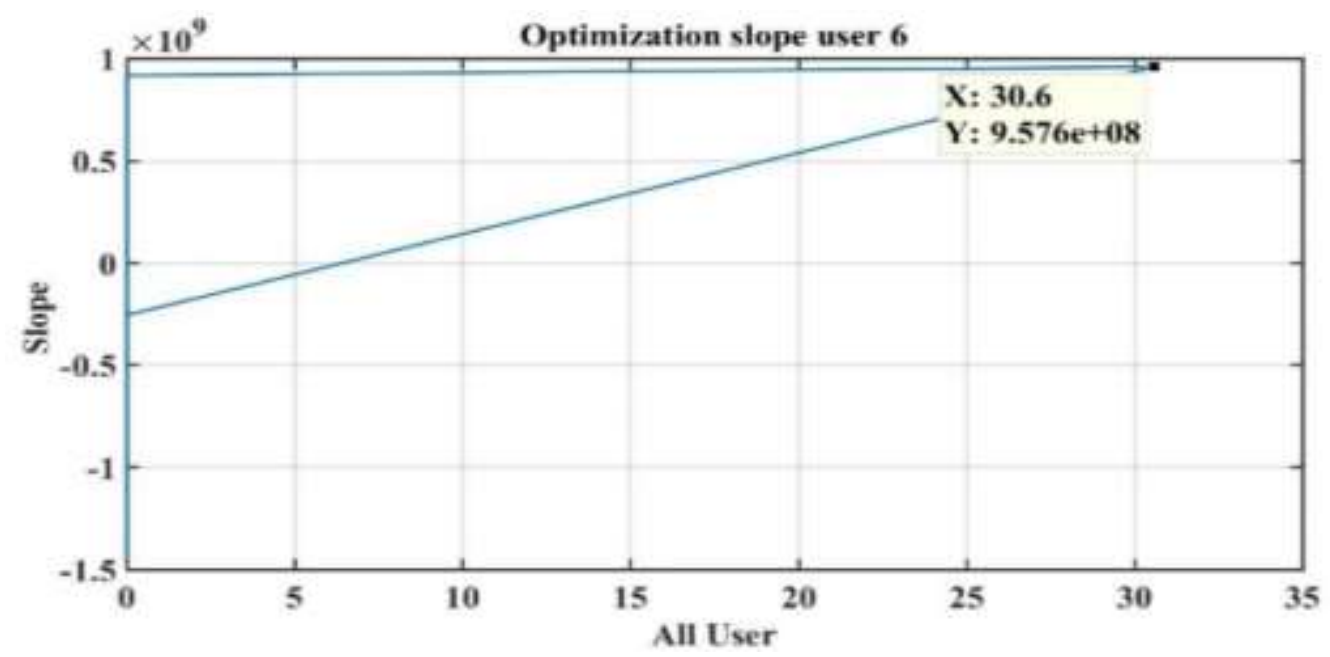

Fig. 16 Optimization Slope for User 6

The optimization slope for user 6 is given by:

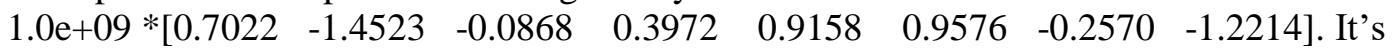
the value of slope(frequency/distance) which is maintained to cancel attenuation due to distance and the optimum value across user 6 is $1.0 \mathrm{e}+09 * 9.576$ then there is no attenuation and exact information of the user can be detected across the receiver. 


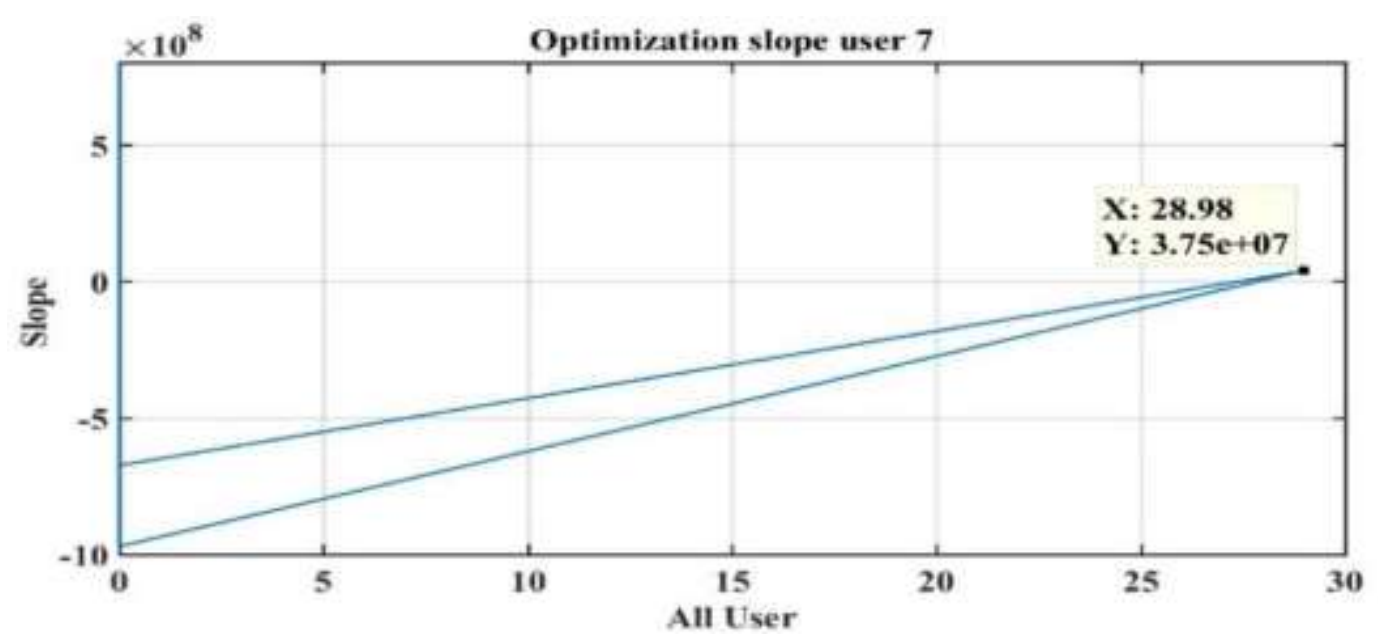

Fig. 17 Optimization Slope for User 7

The optimization slope for user 7 is given by:

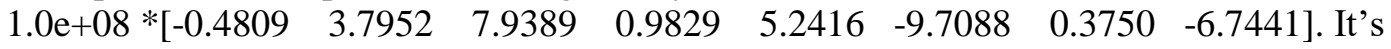
the value of slope(frequency/distance) which is maintained to cancel attenuation due to distance and the optimum value across user 7 is $1.0 \mathrm{e}+09 * 3.75$ then there is no attenuation and exact information of the user can be detected across the receiver.

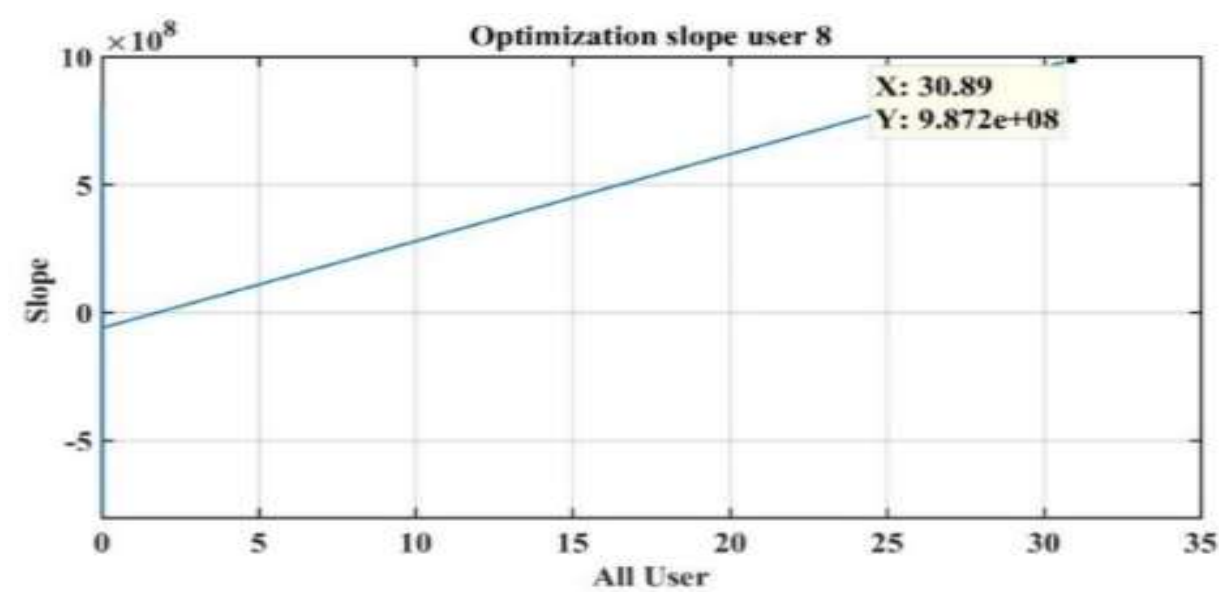

Fig. 18 Optimization Slope for User 8

The optimization slope for user 8 is given by:

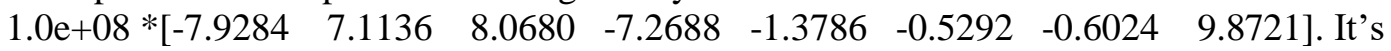
the value of slope(frequency/distance) which is maintained to cancel attenuation due to distance and the optimum value across user 8 is $1.0 \mathrm{e}+09 * 9.872$ then there is no attenuation and exact information of the user can be detected across the receiver.

\section{CONCLUSION}

The proposed model concluded that Adaptive Antenna Technology (AAT) \& Orthogonal Slope Method (OSM) technology will be a good solution for Multiple Input \& Multiple Output (MIMO) technology. The AAT and OSM technology are used to reduce the path loss or attenuation to a much low level where the inverse matrix of the medium is calculated at the receiver and multiplied with attenuation matrix so that attenuation of the medium is reduced. AAT and in OSM technology a slope value(frequency/distance) is maintained so that attenuation due to distance is reduced, if optimum weight or slope value 
is maintained across individual user then there is no attenuation due to the distance which improves the performance of communication system.

\section{References}

[1] F. R. Castillo-Soria, Joaquin Cortez, C. A. Gutiérrez, M. Luna-Rivera, A. Garcia-Barrientos "Extended quadrature spatial modulation for MIMO wireless communications" Physical Communication, (2019), Volume 32, 88-95.

[2] Ratna Chakraborty, Souvik das, Debashis De, "Design of a CDMA transmission reception unit in Quantum dot cellular automata", International journal of computer application (2015),Volume 5,74-84.

[3] Xue Zhang, Cihan Tepedelenlioğlu, Mahesh K. Banavar, Andreas Spanias, Gowtham Muniraju "Location estimation and detection in wireless sensor networks in the presence of fading" Physical Communication, (2019), Volume 32, 62-74.

[4] Naser Al-Falahy, Omar Y. K. Alani "Millimetre-wave frequency band as a candidate spectrum for 5G network architecture: A survey" Physical Communication, (2019), Volume 32,120-144.

[5] Filipe Casal Ribeiro, João Guerreiro, Rui Dinis, Francisco Cercas, Dushantha Nalin K. Jayakody "Multiuser detection for the downlink of NOMA systems with multi-antenna schemes and power-efficient amplifiers" Physical Communication, (2019), Volume 33,199-205

[6] Riyaz Hussain Shaik, Rama Naidu K. "Performance evaluation of dual-hop amplify-and-forward scheme over $\kappa-\mu$ shadowed fading channels" Physical Communication (2019), , Volume 33,206-214.

[7] Muhammet Ali Karabulut, Ozgur Ozdemir, Haci Ilhan "Performance analysis of multiple access relay channels for non-coherent modulations" Physical Communication, (2019). In press, accepted manuscript, Available online

[8] Xu Jiang, Zhendong Yin, Zhilu Wu, Zhutian Yang, Jinlong Sun "Outage probability optimization for UAV-enabled wireless relay networks in fading channels" Physical Communication, (2019), Volume 33, $35-45$.

[9] Aritra De; Tirthankar Datta "Improvement of Performance of MIMO System Using Different Protocols"2018, Fifteenth International Conference on Wireless and Optical Communications Networks (WOCN),(2018), $206-212$.

[10] Aritra De; Tirthankar Datta "Some Aspects of Massive MIMO Spectrum Sharing"2018, Fifteenth International Conference on Wireless and Optical Communications Networks (WOCN).(2018), 1 - 5.

[11] Zhou Fang, Kai Kang, Xiliang Luo, Hua Qian "Performance improvement of a multi-user MIMO system in the presence of nonlinear distortion" Physical Communication, (2018), Volume 31,21-27.

[12] W. C. Y. Lee "Mobile Cellular Telecommunications Systems" McGraw-Hill Inc, Second Edition, 1995.pp 109-114.

[13] J. Yang, Y. Chen, W. Trappe, and J. Cheng, "Detection and localization of multiple spoofing attackers in wireless networks," IEEE Trans.Parallel and Distributed Systems, (2013), vol. 24, no. 1,44-58. 